Itinéraires Itinéraires

Littérature, textes, cultures

2015-1| 2015

Récits de société

\title{
Vie sociale et vie intérieure : la position lyrique dans les sonnets de Gwendolyn Brooks, Pier Paolo Pasolini et Tony Harrison
}

\section{Thomas Vuong}

\section{OpenEdition}

Journals

Édition électronique

URL : http://journals.openedition.org/itineraires/2700

DOI : $10.4000 /$ itineraires. 2700

ISSN : 2427-920X

Éditeur

Pléiade

Référence électronique

Thomas Vuong, «Vie sociale et vie intérieure : la position lyrique dans les sonnets de Gwendolyn Brooks, Pier Paolo Pasolini et Tony Harrison », Itinéraires [En ligne], 2015-1 | 2015, mis en ligne le 14 décembre 2015, consulté le 01 octobre 2020. URL : http://journals.openedition.org/itineraires/2700 DOI : https://doi.org/10.4000/itineraires. 2700

Ce document a été généré automatiquement le 1 octobre 2020.

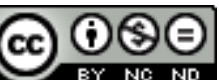

Itinéraires est mis à disposition selon les termes de la licence Creative Commons Attribution - Pas d'Utilisation Commerciale - Pas de Modification 4.0 International. 


\title{
Vie sociale et vie intérieure : la position lyrique dans les sonnets de Gwendolyn Brooks, Pier Paolo Pasolini et Tony Harrison
}

\author{
Thomas Vuong
}

1 Des sonnets, en particulier des sonnets lyriques, semblent de prime abord ne pas constituer le support formel le mieux adapté à la constitution de récits de société ; on imagine mal analyser sociologiquement la bourgeoisie provençale et piémontaise en lisant Pétrarque, ou la cour de Henri II à partir de Ronsard.

2 En tant que forme archétypale du lyrisme occidental - au sens large, depuis William Jones (Essay on the Arts Commonly Called Imitative, 1772) ou les frères Schlegel (Das Gespräch über die Poesie, 1800) d'évocation à la première personne de l'expérience et des sentiments du poète - ou en tant que bref poème articulant différents moments au fil de son huitain et de son sizain, le sonnet semble trop limité pour s'adapter à l'amplitude d'une société entière. Inaugurant la notion de modernité lyrique, Baudelaire, qui a remis le sonnet au goût du jour dans la poésie française, incarne ainsi un regard sur le monde émanant d'un poète en retrait, construit comme à part de la foule qu'il décrit, dans les "Tableaux parisiens " par exemple, ainsi que l'a souligné Walter Benjamin ([1940] 2000 : 357) - et Christian Ruby (2012: 186-192) a pu montrer à quel point ce regard dissocié du monde était également valable dans sa critique esthétique.

3 Et pourtant, chez les trois poètes de la seconde moitié du $x^{e}$ siècle évoqués ici, le prisme lyrique s'articule de manière inattendue avec la description d'une société caractérisée comme un facteur d'altérité; chacun de ces poètes entremêle à une narrativité à la première personne une forme d'auscultation politique et/ou esthétique de son temps, mais qu'il ne se contente pas de regarder, de vilipender ou de déplorer à la manière de la poésie, disons, romantique. Loin du détachement lyrique hérité de Baudelaire, qui pose une distinction entre espaces lyrique et social, ces poètes ont en 
commun d'entrer sur le ring, d'impliquer leur perception intime de la société au sein de celle-ci, de la laisser être remise en cause par ce qu'elle perçoit ; bref, de se présenter non comme des spectateurs hors du monde mais comme des participants à celui-ci.

Le recueil posthume de Pier Paolo Pasolini, L'Hobby del sonetto, publié en 2003 trente ans après la fin de sa composition, est composé de cent-douze sonnets, dont certains inachevés, qui tous abordent une crise amoureuse, celle qui a séparé le poète-cinéaste de son acteur fétiche Ninetto Davoli. Conçu comme une sorte de journal intime entamé en 1971 durant le tournage d'I racconti di Canterbury, l'adaptation cinématographique des Canterbury Tales de Chaucer, ce canzoniere met le poète en rivalité avec la fiancée de Ninetto, qui le ramène vers la vie conjugale. Chaque poème est clairement orienté selon une perspective intime, qui peut être teintée de pathos, de tragique, de satire... Pasolini prend néanmoins soin d'articuler ce lyrisme tout à fait topique, à l'omniprésente première personne, avec les valeurs de la société bourgeoise italienne, qu'il saisit de manière synchronique de façon à constituer un ensemble impressionniste, un archipel, où chaque sonnet apporte une petite précision relative à l'opposition franche qui existe entre des conventions capitalistes et hétéronormées, et l'amour transgressif, univoque et passionné du poète.

Dans ses premiers livres ${ }^{1}$, composés au sortir de la guerre et qui lui ont valu reconnaissance nationale, Gwendolyn Brooks n'a de cesse de revenir au sonnet, que ce soit dans "Gay Chaps at the Bar» (A Street in Bronzeville, 1945) où elle évoque le lien détruit à la vie civile des soldats revenus de la Seconde Guerre mondiale, ou dans Annie Allen (1950) où elle se compose un alter ego, Annie Allen, femme d'intérieur parée des attributs domestiques, que le recueil suit de l'enfance à la maternité. Les poèmes contiennent bien des détails sur la vie de la communauté noire de Chicago aux alentours de la Seconde Guerre mondiale, qui forme un contexte omniprésent plutôt qu'un sujet récurrent des recueils.

6 Plus narrative encore, la séquence de The School of Eloquence de Tony Harrison (1981) évoque de manière autobiographique l'évolution depuis l'enfance du poète, fils d'une famille de la working-class de Leeds, qui devient lettré, apprend l'anglais classique et le latin, et se retrouve étranger à son propre milieu d'origine au moment où ses parents viennent à mourir : la propre valeur de sa parole lyrique est remise en cause par les objets sociaux qu'elle dépeignait. Cette première aliénation trouve un écho dans la description des bouleversements sociologiques et ethniques du Nord de l'Angleterre, où l'ancienne classe ouvrière se voit peu à peu remplacée par des immigrés asiatiques ou caribéens. L'histoire intime s'articule donc avec une histoire sociale des classes populaires anglaises.

7 Quoique de préoccupations différentes, ces trois voix poétiques se trouvent donc impliquées dans le récit de société qu'elles contribuent à construire, à travers la polyphonie, l'inscription de la figure du poète au sein d'un milieu ou d'une idéologie donnés, et plus précisément en raison des rapports tissés entre «je » poétique et rite social.

\section{Polyphonie et perception fragmentée}

8 Disperser les voix constitue un premier moyen de jouer sur le lyrisme, et de l'intriquer profondément dans le récit de société. Cette polyphonie est limitée chez Pasolini : on y lit bien des paroles de Ninetto, de Morante, des citations d'autres œuvres, mais 
digérées, réinterprétées par la voix lyrique. Chez Harrison, c'est principalement la voix du père qui dialogue avec le fils, pour constater le gouffre culturel et social qui les sépare, ou avec les nouveaux résidents de son quartier, qui autour de lui « change plus vite, hélas ! que le cœur d'un mortel », selon le fameux vers du «Cygne » ([1861] 1975 : 85). Brooks, elle, éclate complètement la voix lyrique dans ses sonnets de «Gay chaps at the bar » où chaque poème épouse le monologue intérieur d'un soldat, ou d'une femme restée au pays; dans Annie Allen, elle réunifie le spectre lyrique dans le «je » potentiel d'Annie. Ces délégations de la voix lyrique ne sont évidemment pas nouvelles, et ne valent pas forcément implication. Ce qui me semble notable dans ces micro-récits, c'est que le « je » s'intègre à la société décrite, se situe par rapport à elle.

9 Tony Harrison, ainsi, dépeint une chronique des anciennes classes ouvrières anglaises, et leur remplacement progressif par les immigrants venus des anciennes colonies impériales. Lorsqu'il évoque ses voisins d'enfance, le « old lot » de « Next door », c'est avec leurs habitudes culturelles, telles le goût de la voisine Ethel Jowett pour des troupes d'opérettes comme le D'Oyly Carte ; c'est aussi en donnant, sans le juger, la parole à son père, qui s'inquiète de voir ses voisins mourir et être remplacés par des immigrés :

It won't be long before Ah'm t'only white! (Continuous : 29)

10 Et c'est dans le contraste des pratiques culturelles, vestimentaires ou alimentaires que résident surtout l'incompréhension et la réprobation du père, qui se plaint du reggae qu'écoutent ses nouveaux voisins, s'étonne des tuniques qu'ils portent (et qu'il appelle "sarongs ») ou se désole de ne plus pouvoir acheter de porc ou d'alcool dans les magasins locaux.

11 Au-delà de la simple valeur descriptive de ces poèmes, la voix lyrique s'y trouve doublement impliquée dans les oppositions qui s'y jouent. Tout d'abord parce que la langue du poète est contaminée par les abréviations et les tours lexicaux de l'ancienne working-class du Yorkshire: "for nowt», " $t$ 'fish» au lieu de "the fish», la prononciation de «us » en [uz] à la manière nordique, «mi mam» ou « mi dad » où l'article est déformé, etc. Des termes dialectaux, tel «gob» qui en argot des mineurs désigne le creux laissé par la pépite de charbon après son extraction - et, par extension, la bouche - ou "tusky", terme employé à Leeds pour désigner la rhubarbe, enrichissent également la voix lyrique d'un poète qui prend par ailleurs volontiers Keats ou Wordsworth comme modèles littéraires avoués. Mais c'est surtout parce que le poète, lui aussi, est devenu extérieur à la communauté dont il est issu, qu'il est impossible au « je » lyrique de se prétendre neutre : il est tout autant impliqué dans les liens d'altérité - étranger à ses amis d'enfance devenus piliers de comptoir locaux dans "Divisions", mais aussi, dans "Book ends ", à son père, avec qui il forme une paire déséquilibrée, d'un point de vue sociologique :

The 'scholar' me, you, worn out on poor pay,

only our silence made us seem a pair. (Continuous : 27)

12 Un objet synthétise cette double dynamique d'aliénation : la "flat cap », la casquette plate des ouvriers anglais, qui se fait de plus en plus rarement voir dans la rue, remplacée d'après le père par le spectacle de turbans :

All turbans round here now, forget flat caps! ${ }^{4}$ (Continuous : 32 )

13 Mais la «flat cap » symbolise aussi le gouffre social entre père et fils : lorsque dans «Turns » ce dernier récupère la casquette paternelle, et se plait à s'en coiffer, au motif d'un prétendu gain symbolique : 
I thought it made me look more 'working-class'

(as if a piece of chequered cloth could bridge that gap!) $)^{5}$ (Continuous : 41) bataille européen où est parti l'aimé, ainsi que l'a noté la poétesse Heather McHugh
$(2000)$; c'est par l'altération de la tonalité lyrique, qui se laisse influencer par tout un
imaginaire culturel fantasmé et étranger, que Brooks laisse filtrer le malaise émanant
de la disjonction entre les mondes guerrier, outre océan, et domestique, sur le sol
américain. Les amours d'outre-Atlantique et le mythe du GI américain flirtant avec les
femmes des pays libérés se trouve ainsi vu par l'autre côté de la lorgnette. C'est l'ethos
masculin en tant que tel, fait de violence et de conquête depuis le sonnet initial de
"Gay Chaps at the Bar », qui est épinglé ici. De manière similaire, à la fin de la
séquence, "the progress » témoigne de la désillusion quant aux certitudes patriotardes
de l'Amérique victorieuse:

Il est donc impossible pour le "je " poétique de développer un récit de société sans y trouver des échos de soi-même et de ses propres récits, fussent-ils truqués comme celui de son appartenance à la classe ouvrière.

Gwendolyn Brooks, elle, engage dans ses recueils d'après-guerre le «je » lyrique dans l'opposition aux illusions idéologiques de l'Amérique combattante et conquérante. Dans le «Sonnet-Ballad », elle compose la plainte d'Annie délaissée par son amant fantasmé, parti à la guerre comme dans les bras d'une autre femme :

Some day the war will end, but, oh, I knew

When he went walking grandly out that door

That my sweet love would have to be untrue.

Would have to be untrue. Would have to court

Coquettish death, whose impudent and strange

Possessive arms and beauty (of a sort)

Can make a hard man hesitate - and change. ${ }^{6}$ (Blacks : 112)

La tonalité courtoise de l'allégorie «courtiser / la mort» correspond au champ de
And still we wear our uniforms, [...]
Still we applaud the President's voice and face.
Still we remark on patriotism, sing,
Salute the flag, thrill heavily, rejoice
For death of men who too saluted, sang.
But inward grows a soberness, an awe. ${ }^{7}$ (Blacks : 75)

C'est dans la vie intérieure, opposée par la volta à la vie publique, que se dessine ici la critique émotionnelle de l'émulation martiale ; c'est dans le passage d'une voix lyrique grégaire à sa disparition que se joue le traumatisme des soldats. Ici, la polyphonie atteint ses limites, et Brooks montre à quel point ces voix postiches sont fragiles parce qu'humaines; l'ordre du sonnet, décidé par la poétesse elle-même, joue sur la distribution de la première personne pour mieux raconter le revers de la victoire. Et c'est dans les effets de double sens que la poétesse mine le premier niveau sémantique pour mieux laisser entendre le contraste entre l'héroïsation outrancière du combat, et la réalité domestique une fois revenu: Susan Schweik (1991) a bien analysé les différentes interprétations du mot «bar » dans « Gay Chaps at the Bar », qui au-delà du comptoir où les soldats fanfaronnent renvoie également aux « colour bars », où « bar » a le sens d' " obstacle », des structures racistes en vigueur dans l'armée américaine. Ici, la perception d'eux-mêmes qu'ont les soldats afro-américains est opposée, par la polyphonie poétique, à leur insertion dans une société encore dominée par des séparations fondées sur la couleur de peau. 

opposer vies intérieures et sociales : lorsqu'il dépeint de manière récurrente les lieux interlopes de la vie nocturne romaine, "prato di puttane " (terrain vague à putes", p. 32) ou, au cent-cinquième sonnet, abords de la gare Termini, alors lieu de prostitution masculine, c'est pour s'y représenter en personne et, dans le vingtième sonnet, y situer la rencontre amoureuse, qui est narrée sous le signe de l'altérité. Le poète et son amant se sont rencontrés dans la zone, « tra i rifiuti sparsi sotto quei torrioni ${ }^{8}$ ", c'est-à-dire en dehors des zones balisées de la ville, en dehors des conventions sociales qui y régissent les relations. Le poète va jusqu'à se situer éthiquement dans cette altérité, qu'il renforce par l'obscène, comme celui «chi non ha mai dato a une ragazza il suo seme ", "celui qui n'a jamais donné son sperme à une fille " (L'Hobby del Sonetto : 170). Ailleurs, et à rebours de l'église du contre-modèle pétrarquien, c'est dans un bidonville,

un villaggio di barrache

col suo fango, la sua fontanella ${ }^{9}$

que l'innamoramento a lieu ; ici, c'est le dénuement qui prime, en contraste avec le culte petit-bourgeois de la possession. On voit que la narration est volatile, la cohérence du récit cède le pas à sa valeur allégorique ; ce qui compte, c'est de situer l'amour hors des conventions topiques autant que bourgeoises, et des soucis matériels et sociaux afférents.

Néanmoins ceux-ci ne tardent pas à rejoindre les deux hommes : l'amour homosexuel et spirituel laisse place à une relation, hétérosexuelle et présentée comme matérialiste, entre Ninetto et Patrizia. Leur mariage est même régulièrement réduit à une question financière, voire à un chantage à l'argent ; ainsi, lorsque le poète souhaite que Ninetto le rejoigne sur le tournage durant deux jours,

Ebbene, lei non le ti concede : e giunge a proporti

di mantenerti lei (con le sue cinquantamila

lira al mese ? o con che altro ?)

purché tu le stia sempre vicino ${ }^{10}$.

20 Le résultat du mariage est décrit, de manière particulièrement amère, au sonnet pénultième comme une accumulation de signes extérieurs de médiocrité :

[...] una nera

casa in stile fascista è il tuo nido, un traffico che stordisce in una misera

e presontuosa strada di periferia

è la tua pace $[. . .]^{11}$

C'est le moment où le poète a abandonné la rébellion contre l'ordre établi, ce qui se traduit à nouveau dans la voix lyrique, qui a adopté les points de comparaison de la société bourgeoise. Progressivement, Pasolini se met à quantifier son amour en lires, et à se poser comme élément d'une équation de sexe et d'argent, où la féminité devient une figure du Capital triomphant : ainsi, le sonnet «Richezza? Ah, ah!» se clôt-il sur un vers terrible de renonciation au spirituel, où le poète descend se battre - et, dans la dynamique tragique du recueil, perdre - sur le terrain qu'il estime être celui de sa rivale :

Alla sua ficca oppongo dunque qualche misero milione ${ }^{12}$. 
comme chez Harrison, l'implication du «je » lyrique dans le récit des évolution sociales aboutit à son échec : échec du poète anglais à établir une communication avec son père, aboutissant au silence, et échec du poète italien à résister à l'acculturation capitaliste largement dénoncée dans les Scritti corsari, cette compilation d'« écrits corsaires » où Pasolini analyse les bouleversements brutaux que le capitalisme fait selon lui subir à l'Italie d'après-guerre.

\section{Le poète et le rite}

23 Ces poètes ne demeurent néanmoins pas dans un simple constat d'échec, et lient intimement récit social et première personne poétique au moyen de la notion de rite. Celui-ci ayant pour objectif essentiel d'assurer la cohésion de la communauté, il est évident que le « je » poétique, dès lors qu'il s'inscrit au sein de celle-ci dans un rapport d'altérité, va se trouver rétif, voire en opposition franche avec le rite social.

Pasolini n'a ainsi de cesse d'opposer celui du mariage, à l'union spirituelle, intime, qui selon lui l'a uni à Ninetto des années durant : dans le sonnet « Un mano ha rimestato la profondita ", du cosmos initial naît à la pointe finale l'union naturelle entre deux êtres, qu'aucun rite ne consacre.

Néanmoins, cette intimité du lien est à double tranchant, et le tacite devient tu : au sonnet soixante-et-unième, Pasolini dépeint la déchéance du lien spirituel. De ne pas avoir été prononcé devant les hommes, d'avoir été purement intérieur, l'union que le poète définit comme sa relation avec Ninetto se trouve supplantée par la convention bourgeoise et matérialiste du "stupido anellino", ce risible petit anneau. Le rite spirituel est donc supplanté par une relation sociale, normée :

hai deciso, chissà come e chissà quando,

di vedermi quasi burocraticamente

secondo certe abitudini ormai precise ${ }^{13}$.

Et cette présentation du rite comme vide de sens s'étend à d'autres sacrements: le baptême au sonnet soixantième ou l'enterrement comme union mystique dans la mort dans le sonnet soixante-quatrième. Ici, le rite en tant qu'affirmation d'une norme menace la nature du «je » poétique, que Pasolini se plaît à imaginer duelle - sa part gémelle, celle de Ninetto, lui étant retirée, le «nous» est divisé en «je» et «tu» tiraillés. Diviseur plutôt que rassembleur, source de malheur et d'exclusion pour la voix lyrique en position d'altérité, le rite apparaît comme absurde, son artificialité, sa contingence, parasitent tout à fait l'immanence et la simplicité que le poète perçoit dans leur relation.

27 Au sujet de l'enterrement, Harrison, narrant celui de sa mère dans «Blocks ", met en lumière le caractère proprement aliénant du rite : alors que toute sa famille, qui dans l'ensemble ne comprend guère les préoccupations linguistiques et littéraires du poète, attend de lui qu'il mette à contribution son expérience du langage pour prononcer une oraison, Tony, lui, ne peut que garder le silence - au plus grand dam de ses proches, qui n'en comprennent que moins pourquoi il s'est donné la peine d'apprendre le grec et le latin.

Blocks

A droning vicar bores the congregation 
and misquotes Ecclesiastes Chapter 3.

If anyone should deliver an oration

it should be me, her son, in poetry.

All the family round me start to sob.

For all my years of Latin and Greek

they'd never seen the point of 'for a job',

I'm not prepared to stand up now and speak. ${ }^{14}$

Ici, le rite et les attentes sociales qui s'y nouent préviennent le développement de la voix lyrique, qui demeure dans la sphère de l'intime - à la résolution du sonnet, le poète ne peut que formuler un simple « VALE, MATER » intérieur.

Brooks également se débat contre le rite funéraire, cherche à l'annuler en tant que renoncement à l'intime : dans « the rites for Cousin Vit » (125), l'évocation de la cousine durant ses funérailles se déroule comme si la défunte envoyait elle-même bouler l'atmosphère empesée et les accessoires du cérémonial funéraire : dès le deuxième vers, le sujet de "kicked back the casket-stand («balancé d'un coup de pied le support du cercueil») est élidé, laissant un doute sur qui a commis la maladresse-ou la transgression. Comme le reste du sonnet décrit la vivacité de la cousine Vit, qui semble, aux yeux d'Annie, ne pas pouvoir être durablement mise en bière, l'ambiguïté lexicale semble suggérer que c'est la cousine elle-même, qui à la fin du sonnet dansera et s'agitera, qui entame ici sa révolte contre les derniers rites funèbres en bousculant son propre cercueil. Dans ce sonnet, qui vise à évoquer la défunte à travers ce qu'elle a eu de plus vivant, cette élision initiale sonne comme un refus de la réification du cadavre, ce qui somme toute est la raison d'être d'un enterrement.

Mais c'est surtout la prière qui chez Brooks est l'objet d'un réinvestissement : dans " "God works in mysterious ways" », le « je » changeant des «Gay chaps at the bar » se révolte nettement contre la divinité et, à son point culminant, transforme une prière en ultimatum :

If Thou be more than hate or atmosphere

Step forth in splendor, mortify our wolves.

Or we assume a sovereignty ourselves. ${ }^{15}$ (Blacks : 72 )

L'ultimatum en question concerne directement la place de la première personne, qui menace de prendre la fonction transcendantale de la divinité au cours du rite.

Brooks semble infléchir son aversion, ou tout du moins la nuancer, dans Annie Allen, où la mère, se demandant quelle éducation donner à ses enfants, en vient à récuser dans " And shall I prime my children, pray, to pray? » la validité de la prière, au motif qu'il s'agit d'un rite de soumission à l'ordre donné, qui est celui de la misère :

Children, confine your lights in jellied rules;

Resemble graves; be metaphysical mules; ${ }^{16}$ (Blacks : 117)

À partir de la volta, c'est la mère qui, de façon troublante, assume la prière disqualifiée dans le huitain :

I shall wait, if you wish: revise the psalm,

If that should frighten you: sew up belief

If that should tear: [...]

Holding the bandage ready for your eyes..$^{17}$ (Blacks : 117)

En impliquant la voix d'Annie dans le retournement, l'obéissance finale au rite, en lui faisant formuler l'acceptation de sa nécessité à travers la position de la femme d'intérieur et la métaphore de la couture, Brooks dresse un tableau aigre-doux du 
rapport féminin à la conservation des rites. En dépit de leur inanité, ceux-ci ne sont pas abolis pour autant. tourner le discours, ainsi que la pointe finale, permet aisément de partir d'une donnée initiale pour la développer puis la nuancer ou la mettre en balance avec une autre, de laquelle sortira une pointe conclusive - en cela, la forme du sonnet est liée au processus en mouvement du logos, mais aussi au dessillement, comme dans le sonnet quatrevingt-septième de Pasolini (Sonnets : 180) : «La realtà è un'altra ", à la volta, fait basculer le poème du champ des apparences sociales à celui de la réalité ; l'amour de Ninetto pour Patrizia n'est plus «tutto cio che c'è di bello e la sua convenzione », " tout ce qu'il y a de beau et sa convention ", mais un rapport déchirant d'impossible possession - on notera ici le lien que Pasolini effectue entre société capitaliste fondée sur la propriété, et relations bourgeoises qui en est mimétique.

Gwendolyn Brooks, dont les sonnets sont formellement très classiques, emploie aussi volontiers la volta pour marquer de fortes oppositions : de la formation à la séduction et à la sociabilité, à l'impréparation à la solitude morale dans le premier sonnet de «Gay chaps at the bar "; de ceux qui n'ont pas d'enfants aux parents, dans le premier sonnet de « The Children of the Poor». 
People who have no children can be hard:

Attain a mail of ice and insolence:

Need not pause in the fire, and in no sense

Hesitate in the hurricane to guard.

And when wide world is bitten and bewarred

They perish purely, waving their spirits hence

Without a trace of grace or of offense

To laugh or fail, diffident, wonder-starred.

While through a throttling dark we others hear

The little lifting helplessness, the queer

Whimper-whine; whose unridiculous

Lost softness softly makes a trap for us.

And makes a curse. And makes a sugar of

The malocclusions, the inconditions of love. ${ }^{19}$ (Blacks : 115)

41 Dans cet exemple, comme dans le sonnet "And shall I prime my children, pray, to pray?» cité plus haut, la volta est redoublée par un changement de personne grammaticale, qui passe de la deuxième ou troisième personne à la première. C'est encore une fois la voix lyrique qui se trouve au centre de l'enjeu idéologique du poème, et c'est le sonnet qui donne une forme aux méandres de ses glissements focaux.

Enfin, Tony Harrison, lui, se situe à la marge une fois encore, en choisissant une variante formelle du sonnet, proposée par le poète victorien Meredith dans un recueil de 1862 appelé Modern Love, et composée de seize vers. C'est là faire le choix de ne pas se plier à la contrainte constitutive du sonnet, qui est de composer quatorze vers, tout en acceptant le principe de contrainte: sa position apparait donc éminemment paradoxale, et personnelle. Elle est avant tout motivée par un principe d'élargissement : à l'élargissement du champ poétique opéré à l'intérieur de ses sonnets correspond l'extension des limites de la forme même. En effet, le poète étend systématiquement les limites de la poésie à des objets ou des thèmes qu'elle ignorait jusque-là ; outre, bien entendu, les classes sociales les plus basses de la région de Leeds, c'est un monde entier que Harrison cherche à représenter dans la poésie lyrique - qui était jusqu'au $\mathrm{Xx}^{\mathrm{e}}$ siècle le moins soumis au trivial des genres poétiques. Dans « A Close One" (Continuous: 44), le poète multiplie ainsi les noms de marques de produits consommés durant la période de la guerre, liant le trivial et le souvenir lyrique des choses vécues : les " Messerschmits " menaçants durant le blitz y côtoient les marques de produits vendus dans l'Angleterre des années 1940, tels un "Fry Cocoa's tray", tandis que les cigarettes contiennent des conseils de sécurité, les « Air Raid precautions out of Kensitas ». Avec ce souvenir en particulier, c'est la grande histoire et la petite qui se retrouvent, au sens propre, l'une dans l'autre; comme Proust ou Perec avant lui, Harrison emploie le procédé du dévoilement des différentes strates de la mémoire au moyen d'un petit objet, que la dynamique du sonnet permet d'évoquer, devenant luimême l'image du paquet de cigarettes évoqué :

These lines to hold the still too living dead. ${ }^{20}$ (Continuous : 44)

C'est que le sonnet lui-même est en tant que tel un rite poétique, évidemment: on y sacrifie gratuitement, semble-t-il, et il ne participe d'aucune communauté que de celle des sonnettistes. Rite d'apprentissage pour Gwendolyn Brooks, qui à partir des Bean eaters, où son style arrive à maturité, délaisse la forme ; rite idiosyncrasique pour Tony Harrison, qui déforme le sonnet pour le faire correspondre à l'extension lexicale de la société anglaise ; rite conjuratoire pour Pasolini, qui cherche en vain à contrecarrer les 
fausses bénédictions d'une collectivité artificielle au moyen de la forme des amours interdites - celles de Pétrarque, mais aussi de Shakespeare et Michel-Ange, deux figures tutélaires de sonnettistes de l'homoérotisme. Les récits à l'œuvre empruntent évidemment aux topoï, voire aux clichés, autant qu'à l'autobiographie ; c'est le jeu des poètes de retravailler cette matière brute, de lui donner une forme : ici, quatorze vers un nombre parfois approché par Pasolini, dans certains de ses sonnets inachevés, ou bien porté à seize chez Harrison, dans sa variante meredithienne, tant l'éclatement des visions dans un monde plurivoque entraîne la disparité toujours croissante de formes censément fixes.

Le double caractère dynamique $d u$ sonnet, forme archipélique qui se multiplie en séquences - Tony Harrison a d'ailleurs dit être dévoré par cette sonettomania, pour reprendre le terme que Roubaud (2000: 423) donne à l'attrait irréversible suscité par la forme - et forme progressive qui épouse les voltes de la pensée aussi bien que les péripéties de la narration, me semble expliquer également pourquoi chez certains poètes, il est le lieu privilégié d'une méditation sur le jeu entre « je » et altérité sociale.

Barthes écrivait, en avant-propos de ses Mythologies, qu'elles constituaient une répétition, en forme de recherche de signification éclairant une société étudiée ${ }^{21}$; la répétition du sonnet assemblé en cycles - qu'Edwin Morgan avec les Sonnets from Scotland, William Cliff dans America ou Vikram Seth avec son roman de sonnets The Golden Gate pousseront jusqu'à concurrencer la narration - et sa progressivité dynamique, ses propriétés internes et séquentielles, en font ici une recherche de signification équivalente, dans le domaine lyrique.

\section{BIBLIOGRAPHIE}

Barthes, Roland, [1957] 2007, Mythologies, Paris, Seuil.

Baudelaire, Charles, [1861] 1975, Les Fleurs du Mal, dans Euvres complètes, t. I, Paris, Gallimard.

Benjamin, Walter, [1940] 2000, «Sur quelques thèmes baudelairiens », trad. Maurice de Gandillac et Rainer Rochlitz, dans CEuvres, t. III, Paris, Gallimard, p. 329-390.

Jones, William, ([1772] 1807) «Essay on the Arts Commonly Called Imitative », dans The Works of Sir William Jones, t. X, Londres, John Stockdale.

McHugh, Heather, 2000, « Homage to Gwendolyn Brooks », [En ligne], http://

www.environmentalpress.com/Archive/gwendolyn_brooks.htm, consulté le 12 février 2014.

Roubaud, Jacques, 2000, Poésie :, Paris, Seuil.

Ruby, Christian, 2012, La Figure du spectateur, Paris, Armand Colin. 
Schlegel, Friedrich, 1967, Das Gespräch über die Poesie (1800), dans Kritische Ausgabe seiner Werke, t. II, Munich, Ferdinand Schöningh, p. LXXXVII-XCVI.

Schweik, Susan, 1991, « (Not) Playing with Mimesis. Gwendolyn Brooks and the Stuff of Letters », dans A Gulf So Deeply Cut: American Women Poets and the Second World War, Madison, University of Wisconsin Press, p. 109-139.

\section{Corpus de l'étude}

Brooks, Gwendolyn, [1987] 1991, Blacks, Chicago, Third World Press.

Harrison, Tony, 1981, Continuous, Londres, Rex Collings.

Pasolini, Pier Paolo, [2003] 2012, Sonnets, trad. René de Ceccatty, Paris, Gallimard.

\section{NOTES}

1. Rassemblés dans Gwendolyn Brooks ([1987] 1991) ; je traduis.

2. «Ça ne va pas tarder que j'sois l'seul blanc!»

3. «Moi le "lettré", toi, usé jusqu'à la corde de salaires minimums / seul notre silence nous faisait nous ressembler ».

4. «Que des turbans à la ronde, maint'nant, envolées les casquettes!»

5. «Je pensais qu'elle me donnait l'air prolétaire / - comme si un morceau de tissu à carreaux sur la tête comblaient ce gouffre!»

6. «Un jour la guerre finira, mais, oh, je savais, / Quand il passa flamboyant cette porte / Que mon doux aimé me ferait défaut. / Me ferait forcément défaut. Irait forcément courtiser / La mort, la coquette, dont les bras audacieux et étranges / Étreignent un homme, et la beauté, à sa manière, / Le fait hésiter fût-il solide, et le change. »

7. «Et nous portons toujours nos uniformes [...] / Applaudissons toujours du Président visage et voix, / Commentons toujours le patriotisme, chantons, / Saluons le drapeau, ressentons graves frissons et joie / À l'idée que sont morts d'autres, leurs saluts, leurs chansons. / Mais en nous croît une sobriété, un effroi. »

8. «Parmi les ordures répandues au pied de ces donjons » (Sonnets : 32 ).

9. « un village de bicoques / avec sa boue, sa fontaine publique » (Sonnets : 78).

10. « Eh bien, elle ne te les concède pas, et elle en vient à te proposer / De t'entretenir, elle (avec ses cinquante mille / Lires par mois ? Ou avec quoi d'autre ?) / Pourvu que tu sois toujours près d'elle. » (Sonnets : 186).

11. « une maison noire // De style fasciste est ton nid, / Une circulation étourdissante sur un misérable / Et prétentieux boulevard de banlieue // Est ta paix » (Sonnets : 228).

12. «À sa chatte j’oppose donc quelques pitoyables millions » (Sonnets : 62).

13. « $\mathrm{Tu}$ as décidé, Dieu sait comment, Dieu sait quand,/De me voir presque officiellement / Selon certaines habitudes désormais précises. » (Sonnets : 30).

14. «Blocs // Un vicaire nasillard ennuie la congrégation / Et cite mal l'Ecclésiaste, chapitre troisième. / Si qui que ce soit devait donner une oraison, / Ça devrait être moi, son fils, d'un poème. // Toute la famille autour de moi éclate en sanglots. / D'avoir appris les langues mortes, durant des années / - “à quoi ça sert, pour trouver un boulot ?" - / Ça ne me sert à rien pour me lever maintenant, et parler » (Continuous : 45).

15. «Si Tu es plus qu'haine ou atmosphère, / Avance en gloire, mortifie nos loups. / Ou bien nous assumerons notre propre souveraineté. » 
16. « Enfants, enfermez vos lumières dans des règles gélifiées ; / Ressemblez aux tombes ; soyez des mules métaphysiques ».

17. «J'attendrai, si vous le souhaitez; amenderai le psaume,/S'il venait à vous effrayer; recoudrai la croyance, / Si elle venait à se déchirer; [...]/Gardant le bandage prêt pour vos yeux. »

18. «J'espère que tu les vois sous un meilleur jour !»

19. "Qu'ils peuvent être durs, les gens sans enfants ; / Se confiner dans une cuirasse de glace et d'insolence;/ $\mathrm{Au}$ feu, n'être pas contraints à l'attente; ne voir de sens/Aux hésitations lorsqu'on veille sur un ouragan. / Et lorsque le monde est tout entier mordu, en conflit, / Ils périssent purement, agitant leurs esprits de là, / Sans trace de la grâce, de l'offense, / Qu'il y a à rire ou échouer, emplis de défiance, stellaires. / Tandis que dans une obscurité pétaradante, nous autres entendons / Se lever la petite impuissance; l'étrange / Mélange de couinements et de sanglots, dont la douceur / Perdue ne fait pas tant rire, mais nous piège doucement. / Et devient malédiction. Et fait son miel des / Malocclusions, des inconditions de l'amour. »

20. «Ces vers pour contenir les morts toujours trop en vie."

21. "Je ne sais si les choses répétées plaisent, mais je crois que du moins elles signifient. Et ce que j'ai cherché en tout ceci, ce sont des significations » (Barthes [1957] 2007 : 10).

\section{RÉSUMÉS}

Dans le récit poétique d'une société créatrice d'altérité, ces trois poètes impliquent non seulement leur alter ego poétique dans l'arène sociale, mais mettent en danger leur voix lyrique, qu'une certaine polyphonie sociale peut couvrir ou nuancer. Ils refusent notamment la valeur admise des rites sociaux, qu'ils contestent à la première personne, et proposent à la place un ensemble de rites idiosyncrasiques, qui prennent et que permet la forme du sonnet, qui en vient à canaliser la contestation des poètes et à constituer un rite en soi, sur le plan poétique.

While writing social narratives which set them as outcasts, these three poets not only engage their poetical selves in the social arena but also endanger their personal stances, leaving them exposed to a social polyphony that may either drown them out, or extend their range and depth. They notably use their personal stance in order to challenge the admitted value of social rites, choosing instead to propose idiosyncratic rituals, shaped and permitted by the sonnet form-one that both canalizes the poets' contestation and comes to represent a poetical ritual in itself.

\section{INDEX}

Keywords : compared literature, Brooks (Gwendolyn), sonnet, poetical forms, Pasolini (Pier Paolo), lyricism, rite, community, failure, social life, inner life, (Harrison) Tony

Mots-clés : communautés, littérature comparée, Brooks (Gwendolyn), sonnet, formes poétiques, Pasolini (Pier Paolo), lyrisme, rite, Harrison (Tony), échec, vie sociale, vie intérieure 
AUTEUR

THOMAS VUONG

Université Paris 13, Pléiade (EA 7338) 